\title{
Metodología para la definición de tendencias de aplicación en tecnologías emergentes: Blockchain como caso de estudio
}

\author{
Imanol García-Pastor*, Francisco Sánchez-Fuente**, Jose-Ramón Otegi-Olaso* \\ *Universidad del País Vasco/Euskal Herriko Unibertsitatea, Departamento de Expresión Gráfica y Proyectos de Ingeniería, Bilbao \\ Correo-e: igarcia961@ikasle.ehu.eus | ORCID iD: https://orcid.org/0000-0003-4374-3181 \\ Correo-e: joserra.otegi@ehu.eus | ORCID iD: http://orcid.org/0000-0001-6023-8537 \\ **Universidad del País Vasco/Euskal Herriko Unibertsitatea, Departamento de Organización de Empresas, Bilbao \\ Correo-e: francisco.sanchez@ehu.eus | ORCID iD: https://orcid.org/0000-0002-4558-3452
}

Recibido: 12-04-20; 2a versión: 21-10-20; Aceptado: 27-10-20; Publicado: 20-10-21

Cómo citar este artículo/Citation: García-Pastor, I.; Sánchez-Fuente, F.; Otegi-Olaso, J.R. (2021). Metodología para la definición de tendencias de aplicación en tecnologías emergentes: Blockchain como caso de estudio. Revista Española de Documentación Científica, 44 (4), e310. https://doi.org/ 10.3989/redc.2021.4.1771

Resumen: La velocidad de aparición de tecnologías emergentes se ha incrementado en los últimos tiempos. Esto supone una oportunidad para aquellas organizaciones que las detectan y adaptan de manera temprana, pero una amenaza en el caso en el que sean obviadas por el desconocimiento de su surgimiento. Los primeros signos de aparición de una nueva tecnología derivan de la producción académica, sin embargo, los métodos de detección de dichos indicios se basan en análisis bibliográficos retrospectivos que no permiten identificar de forma directa nuevas áreas de aplicación. Este trabajo desarrolla una metodología para establecer tendencias de aplicación de una tecnología en base a los resultados de análisis bibliométricos. Dicha metodología ha sido contrastada en el concepto de blockchain como tecnología emergente con elevada capacidad de disrupción.

Palabras Clave: análisis bibliométríco; blockchain; vigilancia tecnológica; gestión del conocimiento; innovación.

\section{Methodology for the identification of new trends in emerging technologies: Blockchain as a case study}

Abstract: The rate at which new emerging technologies are appearing has been increasing in recent times. This is an opportunity for those organizations that detect them and adapt early, but a threat in the case where they are overlooked due to unawareness of their appearance. The first indicators of the emergence of a new technology are derived from academic production, however, the methods for detecting these indicators are based on retrospective bibliographic analysis that does not allow the direct identification of new areas of application. This work develops a methodology for establishing trends in the potential applications of a new technology based on the results of bibliometric analysis. Such methodology has been contrasted in the concept of blockchain as an emerging technology with a high capacity for disruption.

Keywords: bibliometric analysis; blockchain; technological surveillance; knowledge management; innovation.

Copyright: ( 2021 CSIC. Este es un artículo de acceso abierto distribuido bajo los términos de la licencia de uso y distribución Creative Commons Reconocimiento 4.0 Internacional (CC BY 4.0). 


\section{INTRODUCCIÓN}

Vivimos en un mundo en el que las nuevas propuestas tecnológicas aparecen cada vez con mayor frecuencia. Esto hace necesario mejorar la capacidad de adaptación de las organizaciones a todos sus niveles, con el objetivo de aprovechar las mejoras que estos cambios pueden aportar a las diferentes necesidades de cada colectivo.

La ingente cantidad de información existente es una ventaja, pero también puede llegar a ser un gran inconveniente si se considera la dificultad de identificar la fiabilidad de las fuentes y calidad de las mismas. Estas complicaciones son todavía más extremas cuando se habla sobre nuevos conocimientos todavía no consolidados en la sociedad.

Las nuevas tendencias, habitualmente, aparecen reflejadas en la producción académica desde sus orígenes. La clave es el poder identificar la información relevante que sirva de base para adquirir nuevos conocimientos científicos en un contexto determinado, y que a su vez impulse la creación de nuevos elementos innovadores. El tratamiento de este tipo de información, para procesarla y convertirla en conocimiento, requiere del tiempo y esfuerzo de personas con amplia experiencia en un sector determinado, y con cierto nivel de influencia si quieren ser empleadas como origen de toma de decisiones.

La gestión del conocimiento (GC) estudia los procesos que llevan al uso eficaz del conocimiento dentro de una organización. Su objetivo es generar conocimiento a partir de información y convertirlo en una ventaja competitiva sostenible que conduzca al éxito de la organización (North y Kumta, 2018). Este valor, denominado capital intelectual (CI), se puede medir utilizando diferentes técnicas. Autores como Bontis (Bontis y Nikitopoulos, 2001), Edvinsson y Malone (Edvinsson, 2013) y Sveiby (Sveiby, 1997) tratan el CI como el indicador de la magnitud de conocimiento que existe en una organización en un momento determinado.

El disponer de una metodología con un alto nivel de automatización, que facilite el análisis de los nuevos documentos publicados dentro de un sector determinado, permitiría incluirla dentro de los procesos de GC. Esto podría minimizar los recursos necesarios para su conversión en conocimiento, y facilitaría su sostenibilidad a lo largo del tiempo. El establecimiento de un análisis metodológico de la documentación publicada, puede resultar muy interesante a la hora de identificar las nuevas oportunidades que brindan e incorporarlas en los procesos de toma de decisiones (López-Robles y otros, 2019). La dificultad sobre la extracción de informa- ción relevante de un modo autónomo es elevada si se tiene en cuenta su formato en leguaje natural.

La propuesta reflejada en este artículo nace de la realización de un estudio sobre nuevas oportunidades de aplicación del concepto blockchain a partir de un análisis de la documentación publicada. Este hecho ha dado como resultado una herramienta de utilización continua que permite realizar una parte de vigilancia tecnológica de un modo automático, permitiendo un enfoque mayor allí donde se detectan aplicaciones de mayor interés o esfuerzo investigador. Al tratarse de una tecnología emergente, en muchas áreas se observó un crecimiento exponencial en publicaciones, comenzando por participaciones en congresos para pasar el testigo más adelante a artículos de revista, generando la necesidad de disponer de mecanismos adicionales que fuesen más allá del análisis retrospectivo y pudiesen generar unas líneas de tendencia de un modo sencillo y eficaz.

Desde su comienzo blockchain ha sido considerada como una tecnología prometedora que puede llegar a ser disruptiva en múltiples sectores (Ahram y otros, 2017). Estas características transversales hacen que su aplicación pueda generar nuevas oportunidades en diferentes escenarios, aunque no de un modo claro. Aplicar blockchain a un área determinada habitualmente implica disrupción, un cambio de modo de hacer las cosas, un cambio de mentalidad, por lo que su utilización va más allá de una mera evolución incremental. Estos hechos hacen que su identificación como tecnología clave y adopción en sectores fuera del financiero no resulte evidente. El disponer de una metodología que permita identificar las ventajas competitivas que este tipo de tecnologías emergentes están aportando a nuevos campos de aplicación es clave. Además, el poder aplicarlo de un modo sistemático posibilita seguir su expansión a lo largo de diferentes áreas, manteniendo la vigilancia actualizada en todo momento.

Actualmente se pueden obtener datos bibliográficos detallados desde diversas fuentes confiables. Como ejemplos se pueden nombrar SCOPUS, Web of Science o Google Scholar. Todas ellas permiten descargar de un modo automatizado diferentes campos de los documentos publicados hasta la fecha, facilitando su posterior análisis (Harzing y Alakangas, 2016).

Este artículo propone la aplicación de una nueva metodología sobre los datos mencionados anteriormente, con el objetivo de extraer de manera sistemática información sobre nuevos campos de aplicación de tecnologías emergentes. Está fundamentada en la combinación de técnicas pro- 
puestas en procesos tales como los empleados en Knowledge Discovery in Databases (KDD) (Fayyad y otros, 1996), Data Science, más en concreto la denominada CRISP-DM (Chapman y otros, 2000), procesado natural del lenguaje y análisis de redes (Zinoviev, 2018). El desarrollo metodológico se ha empleado para analizar blockchain, una tecnología prometedora y disruptiva en muchas áreas, lo que ha permitido obtener de manera objetiva y sistemática el punto en el que se encuentra y sus nuevas áreas de aplicación con mayor potencial.

\section{PROPUESTA METODOLÓgICA}

Los estudios bibliométricos existentes habitualmente están destinados a analizar la literatura científica y a sus autores, utilizando métodos matemáticos y estadísticos, obteniendo una serie de indicadores que parametrizan el proceso investigador. Estos reflejan el número de artículos a lo largo del tiempo, autores, citaciones, factor de impacto y Z-statistic entre otros (Alves y otros, 2016). Durante este desarrollo se ha ampliado este enfoque utilizándolos además para la extracción de nuevas tendencias o aplicaciones en sectores o áreas determinadas.

La realización de este análisis ha tomado como referencia técnica la empleada en 'science mapping' para establecer el proceso del ciclo de vida de los datos, desde su ingesta hasta la producción de información, dejándola lista para la extracción de conclusiones. A lo largo del proceso se ha seguido la metodología CRISP-DM (Cross Industry Standard Process for Data Mining) (Figura 1) adaptándola a las necesidades del estudio (Chapman y otros, 2000). CRISP-DM se aplica ampliamente en el mundo de 'data analytics', estableciendo las fases del ciclo de vida de un proyecto de tratamiento de datos. Una de las ventajas principales de este método frente a otras propuestas es que en todo momento tiene en cuenta las necesidades del negocio, problema u objetivo. En muchas ocasiones llega a formar parte de los procesos de machine learning (Berka y Rauch, 2014), mejorando sus resultados en cada ciclo iterativo y adaptándose a las condiciones del entorno.

Este enfoque ha necesitado de la generación de los algoritmos adecuados para cada una de las fases. Se ha tenido en cuenta el requisito de hacerlos repetibles de un modo automatizado sobre futuros intervalos de tiempo, para poder observar cíclica y continuadamente la evolución y tendencias de la terminología analizada. Como caso concreto de aplicación se ha empleado el término blockchain por sus características, pero su utilización podría extenderse al análisis de otro tipo de tecnologías emergentes o incluso sobre las ya consolidadas.

Figura 1. Metodología CRISP-DM

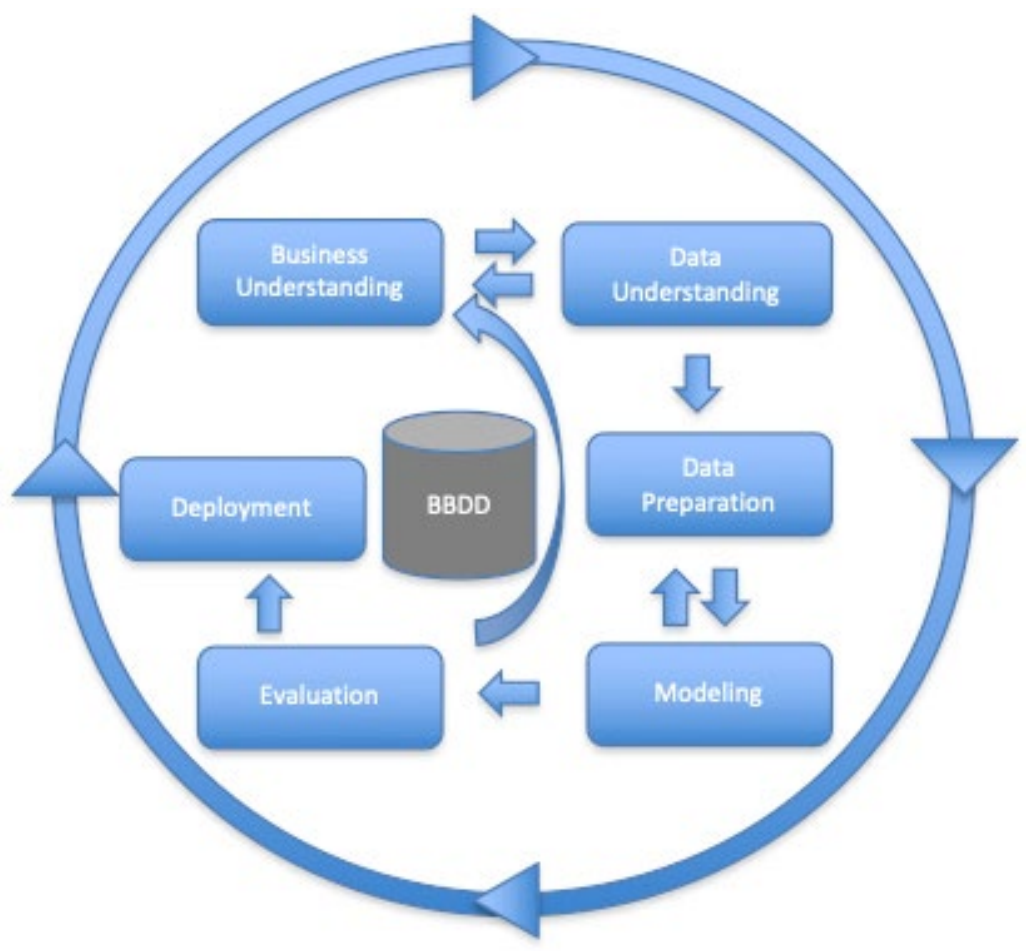


Figura 2. Publicaciones en conferencias y artículos con el término blockchain por año

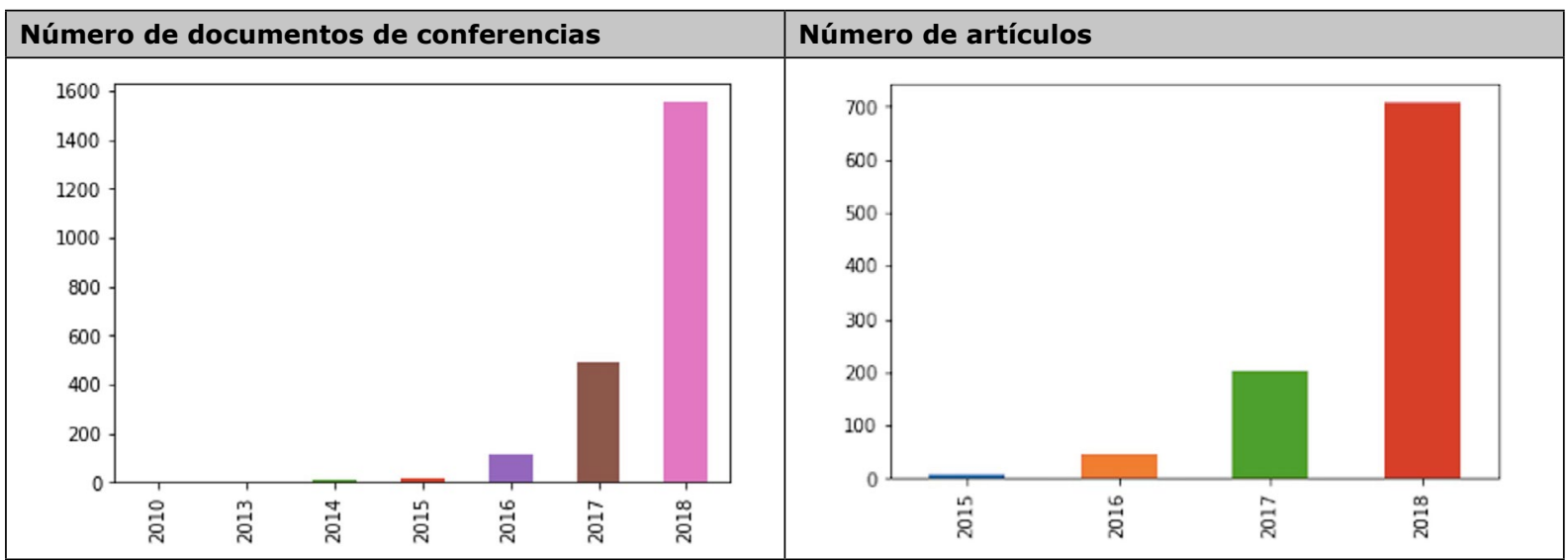

La Figura 2 presenta la evolución del número de documentos presentados en conferencias y artículos de revista a lo largo de los años. El alto número de publicaciones correspondientes a conferencias refleja nuevos desarrollos basados en esta tecnología.

La figura siguiente muestra la expansión de la tecnología blockchain a otras áreas según la cla- sificación de SCOPUS desde el año 2014 hasta el 2018. A lo largo de estos años se aprecia una expansión clara de la tecnología blockchain a diferentes sectores. Este hecho muestra la importancia de realizar una vigilancia dinámica de aquellas tecnologías que pueden resultar interesantes en un área determinada.

Figura 3. Evolución de las áreas temáticas del término blockchain según SCOPUS

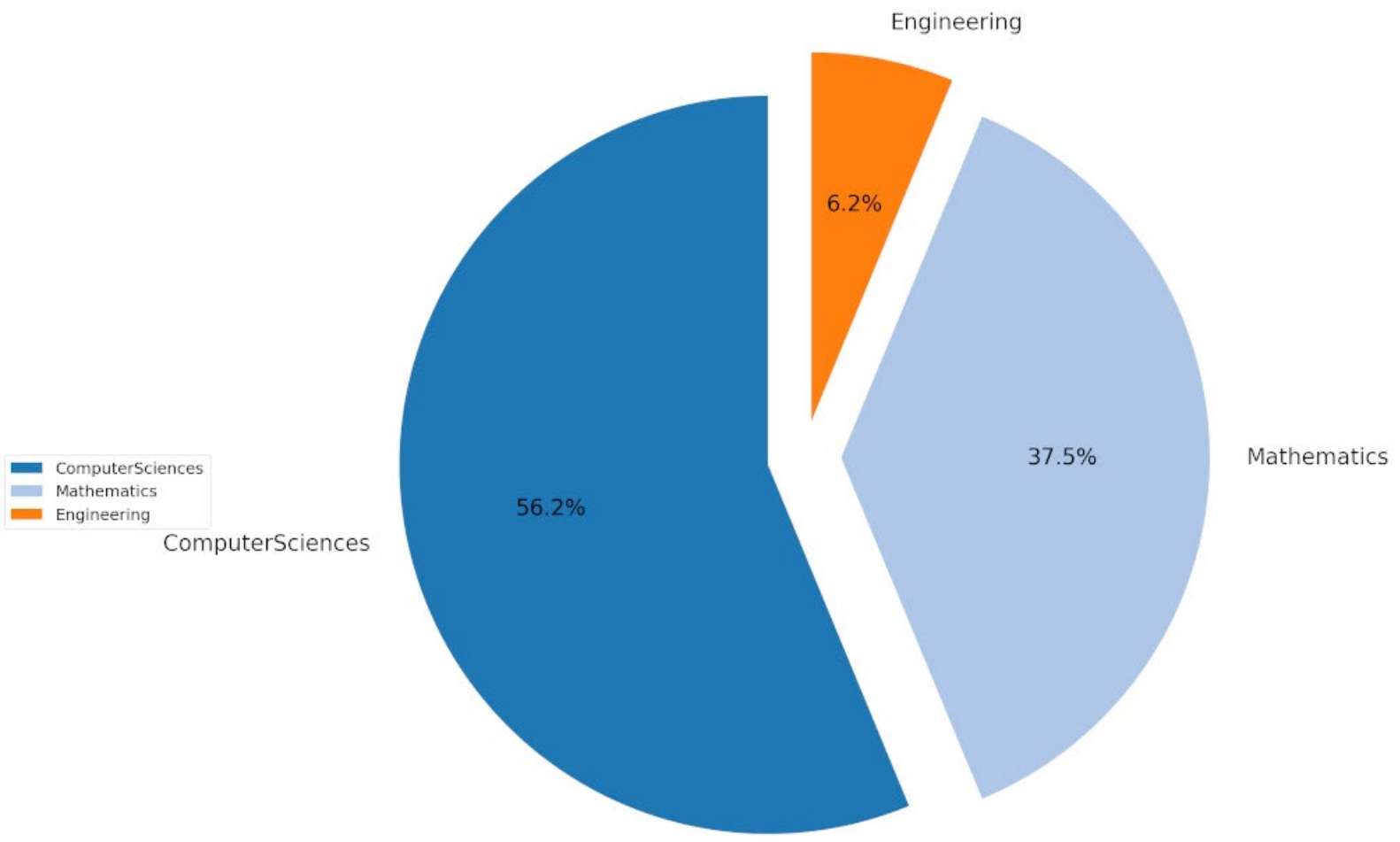


Año 2015
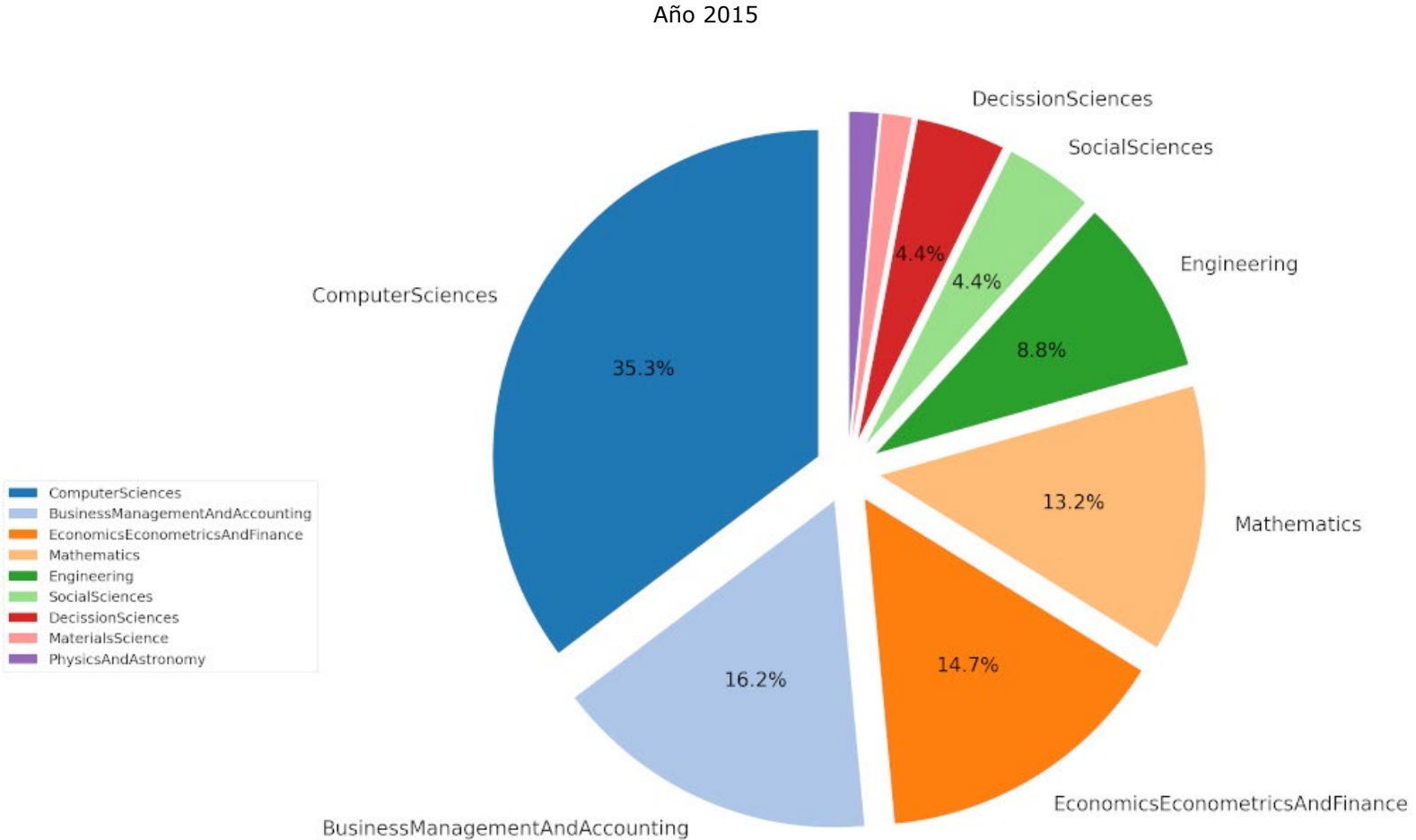

Año 2016
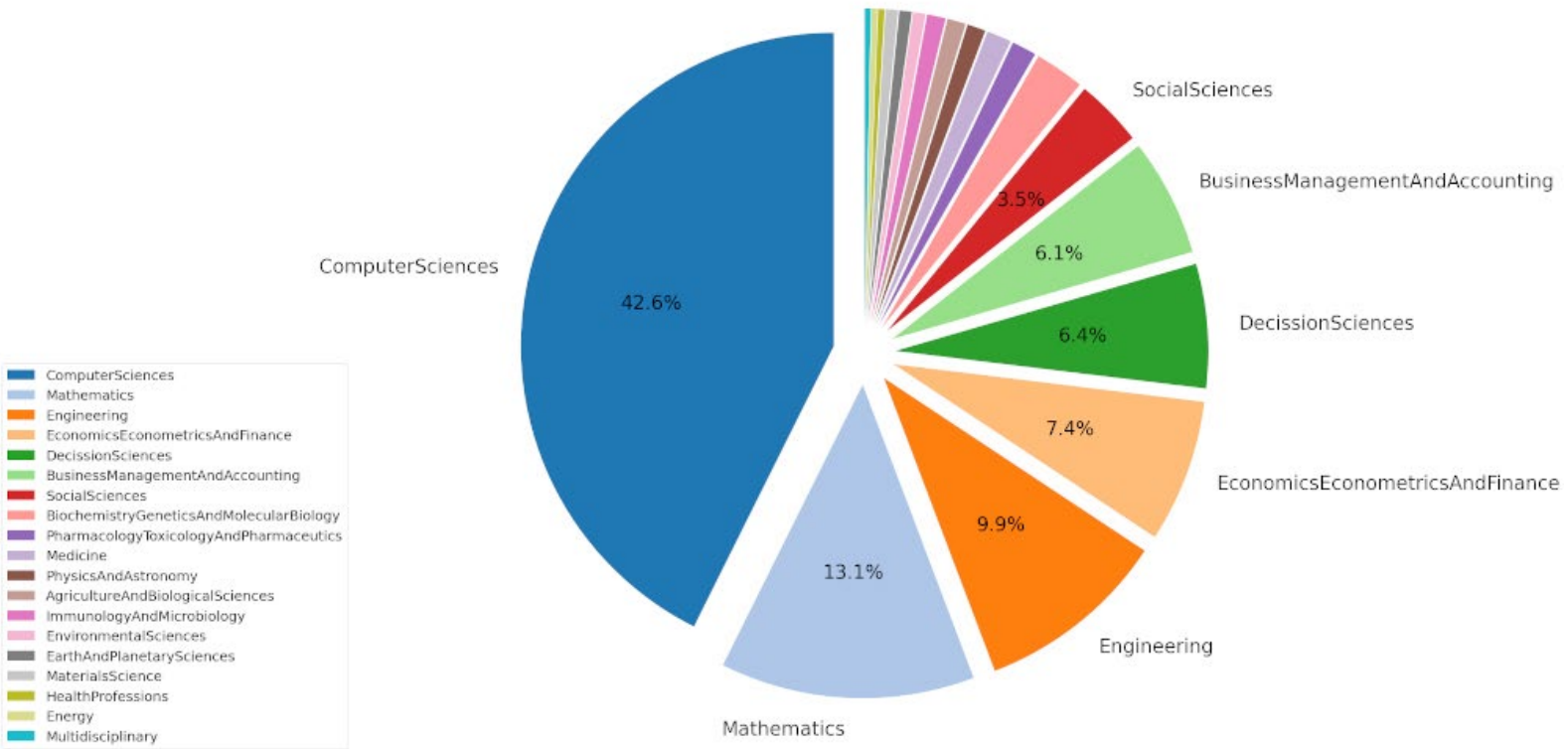
Año 2017
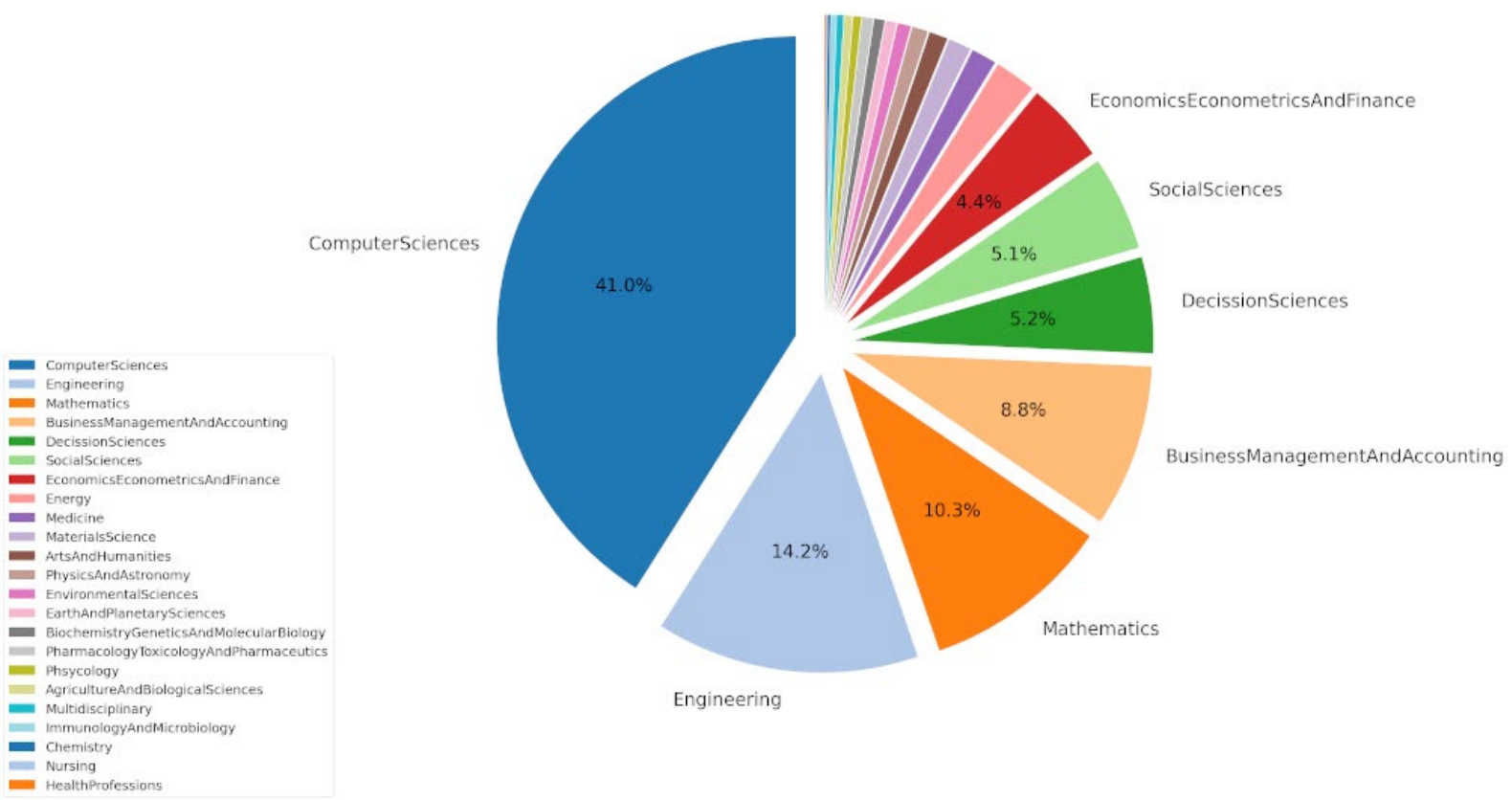

Año 2018

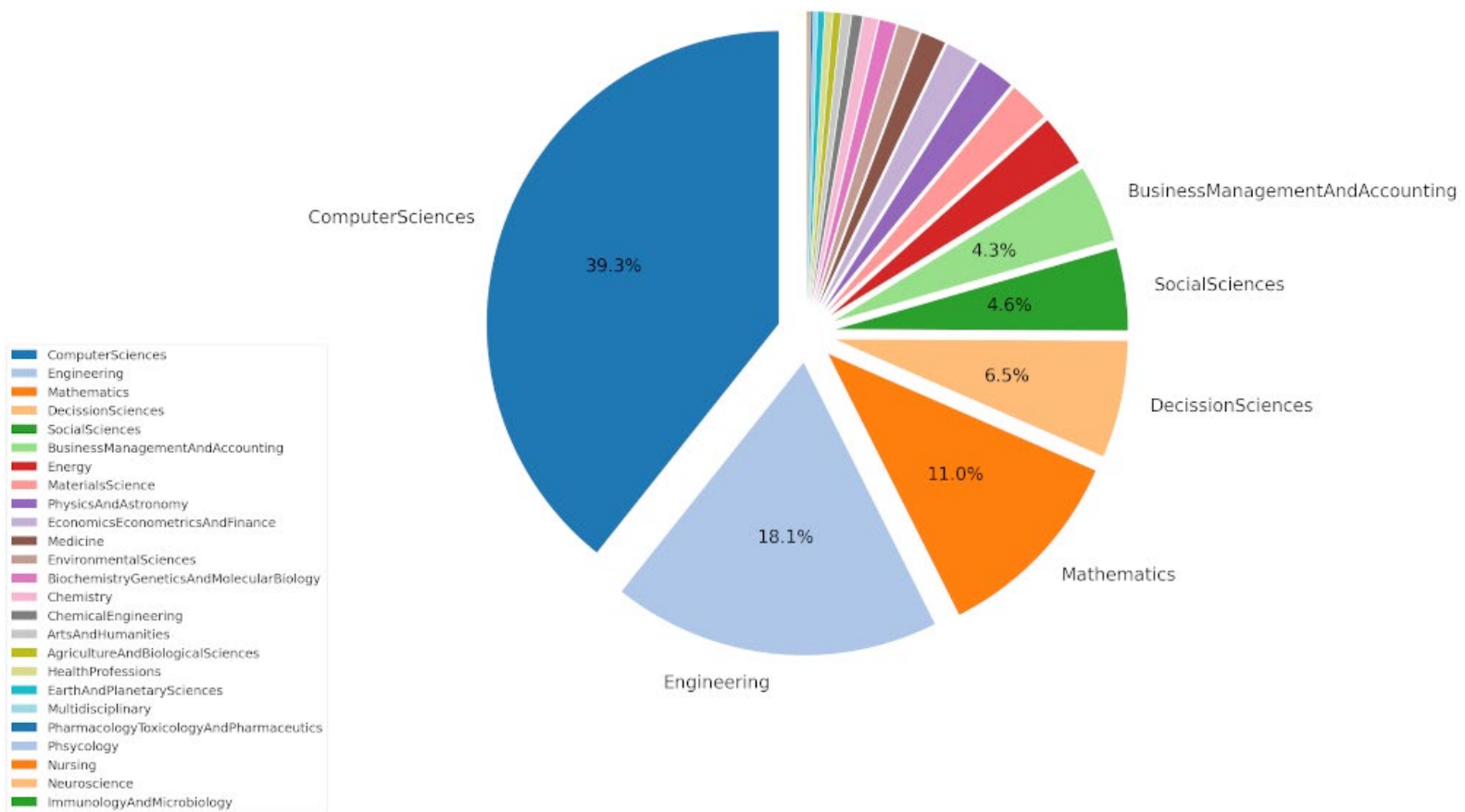


La posibilidad de desplegar los algoritmos de un modo periódico, hace de esta una herramienta interesante desde el punto de vista de gestión del conocimiento y su utilización como activo estratégico. Una monitorización continuada de la información publicada y su posible automatización e inclusión dentro del cuadro de mando de la organización, puede resultar interesante para decidir el momento de invertir o adoptar una nueva tecnología e incluirla dentro de su plan estratégico. Esto es así porque permitiría extraer conclusiones sobre las tecnologías seleccionadas aportando valor de un modo objetivo y único, pudiendo llegar a convertirse en crucial para la toma de decisiones (Michalisin y otros, 1997) (Bollinger y Smith, 2001).

La adaptación realizada se muestra en la figura 4.

A continuación se describen las diferentes fases metodológicas.

\subsection{Fase de definición de objetivos}

Este primer apartado contiene la identificación de los objetivos a conseguir a lo largo de su aplicación. Tal y como se ha comentado en el punto anterior, la metodología puede tener como meta el convertirse en uno de los procesos claves de vigilancia de las tecnologías emergentes que afectan a un negocio determinado. Para que esto sea operativo se ha considerado que la implementación propuesta debe de ser:

- Automática: sin intervención humana o con la menor posible. Sin sobrecargar los procesos ni añadir un coste significativo.

- Objetiva: en base a información confiable y concreta extraída de los registros bibliográficos.

- Fácil de usar: generando indicadores interpretables de un modo sencillo e integrables en un cuadro de mando.

- Capaz de mejorar y aprender: a través de la mejora de los algoritmos integrados y pudiendo evolucionar a un sistema de 'machine learning'.

Los resultados obtenidos deben de reflejar las tendencias y evolución de una nueva tecnología, permitiendo identificar sus ventajas en un área determinada y adoptarla cuando se considere un elemento suficientemente maduro y competitivo.

Como indicadores principales se propone tener en cuenta los siguientes:

- Identificación de áreas temáticas.

"Análisis relacional.

» Índice de expansión.

- Identificación de áreas de aplicación

»Análisis relacional.

» Índice de expansión.

Figura 4. Esquema de la Propuesta Metodológica

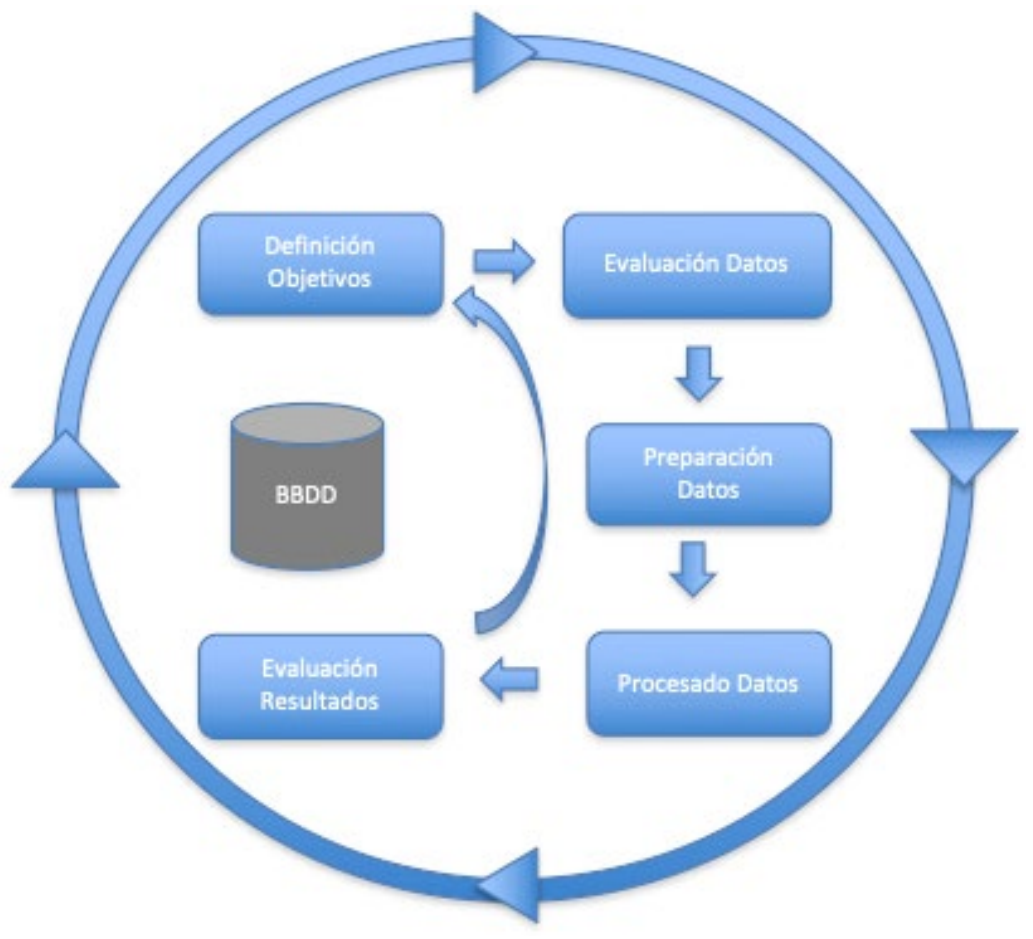


El primero de los puntos está orientado a detectar la expansión de la tecnología analizada en temáticas o campos en donde no es tan habitual su utilización. El segundo pretende identificar los casos principales y concretos de aplicación. Cada indicador se construye a través de un análisis relacional y una estimación del factor de expansión en cada una de las áreas.

\subsection{Fase de obtención de los datos}

Como origen de información inicialmente se han tenido en cuenta tres fuentes de bases de datos bibliográficas ampliamente utilizadas: Web of Science (WoS), SCOPUS y Google Scholar. Todas ellas se han considerado válidas para el tipo de estudio que se ha llevado a cabo (Aguillo, 2012) (Harzing y Alakangas, 2016). Los factores que han primado a la hora de elegir una u otra han sido principalmente los mostrados a continuación:

- Número de documentos reportados a la hora de realizar la búsqueda: al pretender utilizar los datos de las publicaciones para extraer conclusiones más allá del análisis bibliográfico, se ha optado por la fuente que permite descargar datos de un mayor número de documentos.

- Facilidad del tratamiento de los datos extraídos para su posterior procesamiento: la homogeneidad y el formato de los datos puede facilitar su análisis. WoS y SCOPUS permiten exportar sus registros en formatos estándar tales como 'coma separated values' (CSV).

- Número de campos por registro: poder disponer dentro de los registros de un mayor número de campos de información, habilita nuevas posibilidades dentro del desarrollo de los algoritmos. Llegando a mejorar su fiabilidad y capacidad de extracción de conclusiones.

En este punto ya se identifica la necesidad de adaptación de los datos en los registros CSV. Por un lado, para convertirlos en una base de datos más manejable programáticamente $y$, por otro, para normalizar las expresiones no homogéneas en la parte de las palabras o términos clave.

\subsection{Fase de preparación de los datos}

Este apartado incluye la conversión sistemática de los datos descargados a una estructura tratable por los algoritmos a emplear en la siguiente fase de procesado y evaluación de los mismos. Al tratarse de un formato de texto en CSV, hace falta que su procesado sea homogéneo y no dependa de caracteres que puedan dañar los pasos posteriores. Para ello existe una etapa inicial de filtrado y homogeneización del texto. Después, se incluye en los registros la información de las temáticas que abordan cada uno de ellos según SCOPUS.

Uno de los campos que se han tenido en cuenta es el apartado de palabras clave o 'keywords' de autor de cada documento. Este hace referencia a las palabras o conceptos de destacable importancia dentro de un texto, debiendo representar el contenido del mismo siendo a su vez específicas en el contexto tratado. Aportan, por lo tanto, información relevante condensada en pocas palabras. El principal problema reside en que son cadenas de texto, por lo que el mismo concepto puede expresarse de diferentes maneras.

Al estar tratando de procesar lenguaje natural se propone emplear mecanismos provenientes de esta área (NLP en inglés) (Xi y Cho, 2012) tales como el estemizado o el lematizado, orientados a la obtención de la raíz de los términos y su parte canónica respectivamente.

La salida de esta fase aporta una serie de registros procesables de los cuales se pueden extraer detalles sobre la evolución de la tecnología analizada a lo largo del periodo considerado.

\subsection{Fase de procesado de los datos}

En este apartado los datos se someten a diferentes análisis, generando una serie de resultados orientados a dar respuesta a los puntos planteados en la fase de definición de objetivos. Éstos, en su forma numérica y gráfica, serán interpretados durante la fase de evaluación.

La naturaleza de los datos tratados, así como los objetivos planteados, hacen que el empleo de análisis de red sea una aproximación adecuada. Las diferencias principales de este estudio frente a otros basados en datos bibliográficos residen en su apoyo en este tipo de técnicas. Los análisis de red permiten representar entidades y las relaciones entre ellas (Zinoviev, 2018). Esto ayuda a centrar y extraer información relacional de importancia entre las diferentes temáticas propuestas por SCOPUS, o identificadas analizando las palabras clave de autor presentes en los artículos.

Se han utilizado conceptos de teoría de grafos para extraer conclusiones de los diferentes tipos de agrupamientos y relaciones entre variables. La descripción de las diferentes redes se ha realizado mediante matrices de adyacencia. Una matriz de adyacencia se puede definir como una matiz de dimensiones $\mathrm{NxN}$, siendo $\mathrm{N}$ el tamaño del grafo a representar y teniendo por filas y columnas los nodos origen y destino respectivamente (Zinoviev, 2018). Indicadores como el índice de agrupamiento (clustering coefficient) o grado de centralidad 
(degree centrality) y su evolución a lo largo de los años, o respecto a áreas geográficas, muestran el posicionamiento de las diferentes características analizadas.

Dentro de la teoría de grafos y análisis de red, hay varias medidas de centralidad (centrallity) de un vértice que determinan la importancia del mismo dentro del gráfico. Este nivel de importancia se puede llegar a contextualizar, pudiendo alcanzar conclusiones específicas sobre los conceptos analizados.

Existen cuatro medidas de centralidad utilizadas de un modo muy amplio. Referidas por sus nombres en inglés son las siguientes (Opsahl y otros, 2010):

- Degree Centrality: se denomina al número de enlaces que conectan ese nodo respecto al número total. Este índice posiciona los diferentes nodos por su número de conexiones con otros. Dependiendo del contexto puede indicar popularidad.

- Betweenness Centrality (Cb): es el índice que muestra cómo de centrado se encuentra un vértice centro de un grafo de red. Se calcula obteniendo para todas las parejas de nodos los caminos más cortos entre ellas, determinando que fracción de ellos pasan por el nodo en cuestión y sumándolos todos. En el caso de tener un grafo de estrella con un elemento central y el resto nodos adyacentes, el índice de $\mathrm{Cb}$ sería 1 , normalizado para el nodo central, y 0 para el resto.
- Closeness Centraility: indica los nodos con los caminos más cortos, dando un valor mayor para los vértices centrales. Conceptualmente suele relacionarse con la velocidad con la que fluye la información u otras características de un vértice a otros.

- Eigenvector Centrality: mide la importancia de un nodo dentro de la red. Asigna puntuaciones relativas a todos los nodos de red basándose en el principio de que los nodos con mayor puntuación contribuyen más a sus conexiones que los de menor ranking. Aplicaciones como 'Google Pagerank' utilizan variantes de esta medida.

'Eigenvector Centrality' es un buen indicador para conocer el ranking de los nodos de una red según su nivel de influencia. Esta técnica se utiliza desde mediados del siglo XX en análisis sociales y grupales.

Con el ánimo de dar respuesta a los indicadores seleccionados previamente, este proceso se ha realizado en dos pasos: en primer lugar, aplicándolo a las áreas temáticas propuestas por SCOPUS, y después a las palabras clave de autor.

A continuación, debido a que la dependencia de las áreas base en el caso de análisis de tecnologías emergentes puede no permitir distinguir agrupaciones (clústeres) claras, se propone la división en conjuntos relacionales en base a las palabras clave de autor con una mayor importancia en base al índice Eigenvector aparecido en el análisis global.

Figura 5. Metodología de Identificación Áreas BC: Procesado de los Datos (Figura 4)

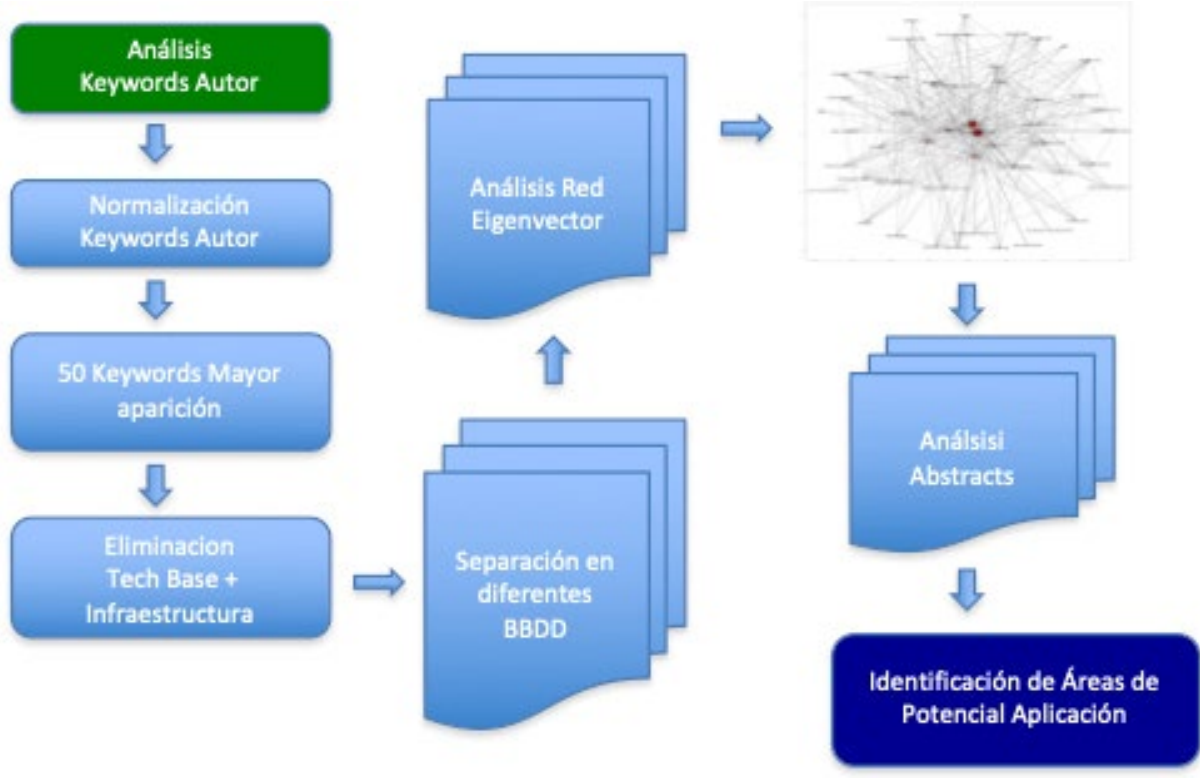


El proceso completo de tratamiento de los términos clave de autor se muestra en la Figura 5.

Esto permite profundizar en el conocimiento sobre las áreas de mayor desarrollo de la tecnología objetivo y además realizar un zoom dentro de cada una de ellas, volviendo a aplicar un análisis de red de los subconjuntos de documentos que contienen ciertas palabras clave relacionadas con una temática en concreto.

\subsection{Fase de evaluación}

Este apartado se centra en la evaluación de los datos obtenidos para dar respuesta a los objetivos planteados. Esto lleva a la interpretación de los resultados obtenidos en el punto anterior sobre los datos utilizados sobre la tecnología.

En primer lugar se realiza un análisis de temáticas o áreas de aplicación. Los datos obtenidos referentes al volumen de publicaciones por temática y tipo de publicación, mostrará el punto de desarrollo en el que se encuentra por áreas y, de su evolución, si se dispone de un histórico de datos anterior en el caso de estar ya desplegada la metodología. Además, los indicadores como el índice de centralidad Eigenvector y la representación de su análisis de red, son dos magnitudes propuestas para su integración en el cuadro de mando.

En segundo lugar se propone utilizar las conclusiones extraídas del análisis de las palabras clave de autor normalizadas. De ellas se extraen, por un lado, los términos de mayor relevancia según su índice de centralidad. La separación en subconjuntos liderados por los términos con mayor Eigenvector para después realizar un análisis de red de los mismos, presenta no solo las nuevas áreas de aplicación en un área determinada sino, también, cómo se están proponiendo utilizar con casos concretos. En este caso los indicadores seleccionados son el índice de centralidad Eigenvector de los términos normalizados y los análisis de red realizados sobre cada subconjunto.

\subsection{Despliegue de la metodología}

Se considera despliegue el hecho de incorporar los mecanismos propuestos a los procesos de la organización, realizando el análisis de modo iterativo y convirtiendo los resultados de la evaluación en un indicador o conjunto de indicadores que permitan mejorar el nivel competitivo de la misma.

Vivimos en un entorno cambiante y de evolución constante, en donde la innovación, la adopción de nuevas tecnologías y su inclusión dentro de modelos de negocio es una necesidad para no quedarse descolgado de las tendencias de mercado. Del mismo modo, es imprescindible detectar la obsolescencia de los elementos tecnológicos empleados con una suficiente antelación que permita su sustitución.

La herramienta propuesta y descrita está pensada para activarla de manera periódica sobre una o varias tecnologías que pueden afectar a un sector en concreto, y sus salidas pueden emplearse como indicadores además de como entrada a procesos de generación de nuevos modelos de negocio o mejora de los ya existentes.

La monitorización periódica de los indicadores propuestos permite identificar las áreas de influencia de una nueva tecnología a lo largo del tiempo a través de dos aproximaciones diferentes: temáticas e identificación de tipos de aplicación. De este modo se puede seguir, además, su evolución para poder determinar el momento más interesante para comenzar su adopción o apostar por una nueva aplicación emergente. Siendo de utilidad tanto para innovaciones incrementales como disruptivas y encajando en los diferentes modelos de este término que han aparecido a lo largo del tiempo (Kogabayev y Maziliauskas, 2017). La disposición del valor numérico de centralidad Eigenvector y su posible interpretación facilita su uso como indicador dentro del cuadro de mando.

El despliegue de este nuevo elemento, que puede considerarse dentro de las herramientas de vigilancia tecnológica, se encontraría en la fase inicial de las propuestas a desarrollar dentro de los procesos de innovación de la organización tal y como se muestra en la Figura 6.

En el caso de blockchain, la identificación de sus posibles usos y el conocimiento de sus nuevas áreas de aplicación puede indicar la necesidad de incluirlo en nuevos productos o servicios en un sector determinado. El amplio espectro de uso de esta tecnología y su gran potencial hace muy necesario un seguimiento cercano de su evolución dentro del sector donde desempeña su labor una organización concreta.

\section{DISCUSIÓN}

La calidad y riqueza de los datos es indispensable para obtener conclusiones correctas. SCOPUS dispone de la posibilidad de descarga de registros detallados con información de cada documento. Además, clasifica internamente los mismos según áreas temáticas propuestas por la propia web, lo cual permite enriquecer los datos con esta nueva característica. Este tipo de técnicas destinadas a añadir nuevos detalles a la información inicial a través de su contextualización se denominan 'Feature 
Figura 6. Integración dentro del proceso de innovación

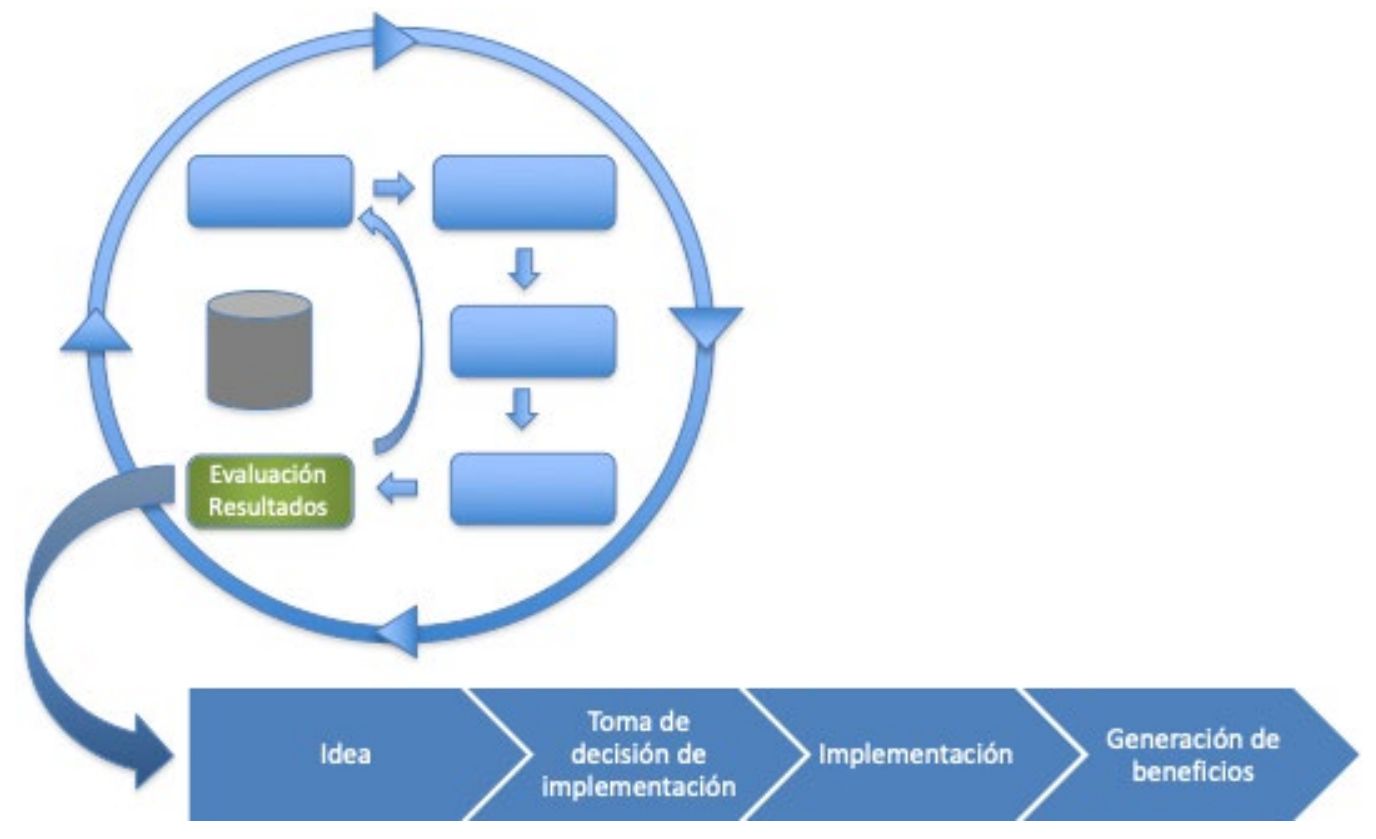

Engineering' y pueden poner en relieve importantes detalles a la hora de alcanzar los objetivos fijados. En este caso en concreto supone una orientación inicial sobre el área de aplicación de cada documento. Al tratarse de una clasificación interna de SCOPUS, hay que tener en cuenta que puede añadir cierta subjetividad, pero facilita la obtención de una primera impresión sobre la evolución por áreas de la aplicación de la tecnología o término analizado. Cabe destacar que un mismo artículo puede pertenecer a varios subconjuntos temáticos.

Los campos de los que dispone SCOPUS para cada artículo son muy variados y ricos. A lo largo de este estudio se ha utilizado principalmente los datos de título, fecha, palabras clave de autor y temática. Queda así un amplio margen de análisis y mejora al poder incorporar información que resulte también significativa como lo son las citaciones o los 'abstracts'.

Para mejorar y afinar los resultados del análisis, es necesario pasar por un proceso de normalización que permita agrupar palabras clave independientemente de cómo se escriban. Esto se complica aún más en diversas ocasiones, como ocurre por ejemplo cuando una palabra clave está formada por varios vocablos, se trata de términos técnicos compuestos, o expresan conceptos que no están aún estandarizados en la temática. Además, hay que tratar las diferencias de letras (mayúsculas/ minúsculas), géneros, plurales o los posibles errores de deletreo entre otros aspectos.
A pesar de todos los esfuerzos, en muchas ocasiones una parte de las palabras analizadas necesitará tratamiento manual si pretenden ser incluidas en el estudio. En cada caso concreto habrá que considerar si el proceso automatizado es suficiente para disponer de una muestra significativa que permita extraer resultados concluyentes y certeros.

Las técnicas aplicadas de clasificación y agrupación conceptual basadas en análisis de red permiten extraer conocimiento durante la fase de evaluación. Estas pueden automatizarse fácilmente para integrarlas en el despliegue de la metodología, instalándolas dentro del proceso propuesto para ser aplicado periódicamente. De este modo pueden utilizarse como indicadores KPI dentro del cuadro de mando, implementando un mecanismo de vigilancia periódica automatizada de las áreas tecnológicas que pueden ser de gran interés estratégico y de negocio.

Dentro de las tecnologías emergentes, el empleo de técnicas de agrupación puede no llevar a conclusiones debido a su alta dependencia de las tecnologías base sobre las que parten. Sin embargo, la separación en conjuntos relacionales basándose en las palabras clave de autor con un mayor índice Eigenvector permite discernir la evolución de las aplicaciones en un área determinada.

Como caso de estudio se ha empleado la tecnología blockchain, nacida en 2008 y con una expansión y potencial en muchos sectores más allá 
del financiero (Ahram y otros, 2017). Esto se ve reflejado en su evolución a lo largo de los años (Figura 3), con un número de aplicaciones novedosas que llegan a ser disruptivas en algunas ocasiones. A continuación se muestra la aplicación de la metodología y los resultados sistemáticos obtenidos.

\subsection{Conjunto de datos extraídos de SCOPUS: término 'blockchain'}

Al tratarse de un concepto nuevo, la búsqueda de la palabra 'Blockchain' dentro del título, del abstract o de las palabras clave, desde el 2000 hasta el 2018 (TITLE-ABS-KEY (blockchain) AND PUBYEAR > 1999 AND PUBYEAR < 2019), no retorna un número elevado de ocurrencias en el momento de su realización el 28-04-2019: 3375 documentos.

Estos resultados pueden ampliarse complementando la búsqueda con otro tipo de términos relacionados con esta tecnología, como pueden ser aquellos que se encuentran en su base conceptual (DLT, distributed ledger...) o fundamentos tecnológicos (Merkle Tree, consensum algorithms...). No se han añadido porque pueden aumentar la dificultad de sistematizar el proceso y añadir ruido a los datos iniciales debido a que podrían aparecer documentos no relacionados con el término principal. Sí que sería interesante tenerlos en cuenta en futuras evoluciones de la metodología propuesta, pudiendo ser de gran ayuda en el estudio de tecnologías más consolidadas.

La recogida de estos datos se realizaría de un modo periódico, añadiendo a la entrada del proceso conjuntos de datos que incorporen las nuevas publicaciones surgidas. El disponer de datos actualizados a lo largo del tiempo pone a disposición del análisis datos evolutivos y dinámicos que pueden mostrar detalles significativos tales como tendencias de crecimiento y velocidad de adopción.

\subsection{Preparación de los datos: término 'block- chain'}

Los datos descargados necesitan de un tratamiento previo antes de ser procesados. Los pasos dados a lo largo de este proceso han sido los siguientes.

1. Filtrado y homogeneización del texto en CSV.

2. Procesos de 'Feature Engineering': enriquecimiento añadiendo información complementaria. Este es el caso de la temática según SCOPUS.

3. Palabras clave: normalización del texto de los términos.

4. Palabras clave: normalización utilizando NLP.

5. Modificaciones manuales si se considera necesario.
Esta fase dispone de un amplio desarrollo futuro. Durante el momento del preprocesado de los datos se pueden añadir características a los mismos que pueden permitir más adelante extraer importante información. Técnicas como las empleadas en minería de datos o procesado natural del lenguaje se pueden añadir en esta fase para mejorar los resultados posteriores e incluirlos en posibles futuros algoritmos de aprendizaje automático incorporados a la metodología. En este caso, se ha empleado únicamente para añadir el campo de temática a cada uno de los registros. Esto ha permitido representar la evolución temporal de las áreas en donde se está explorando de manera activa las oportunidades de la tecnología blockchain. Desde su comienzo ha sido identificada como una tecnología prometedora e incluso disruptiva en multitud de sectores. Sin embargo, su aplicación y maduración dentro de cada uno de ellos sigue sin ser del todo clara.

A pesar de las grandes posibilidades de esta tecnología (Boucher y otros, 2017) (Chartered Accountants, 2016) (Dr Garrick Hileman y Michel Rauchs, 2017), su aplicación no siempre es evidente. Esto es debido a que su empleo en muchas ocasiones no presenta mejoras incrementales, sino que define nuevos modos de actuación, lo que hace necesario superar el miedo al cambio. Además, hay que tener en todo momento presentes sus limitaciones (Conte de Leon y otros, 2017).

En el 2018 la diversidad de áreas en donde existen publicaciones sobre blockchain ha seguido aumentando exponencialmente (Figura $\mathbf{3}$ ).

\subsection{Procesado de datos en el conjunto extraí- do del término 'blockchain'}

Durante esta fase el objetivo tenido en cuenta en todo momento es la identificación y monitorización de las áreas de aplicación de la tecnología blockchain, enfatizando aquellas que son diferentes a las más conocidas como pueden ser las financieras. Para ello se utilizan los análisis de red y sus datos asociados, como el índice de centralidad Eigenvector, para la obtención de conclusiones e indicadores. Dichos análisis se aplican sobre las áreas temáticas y las palabras clave de autor previamente normalizadas.

\subsubsection{Temáticas SCOPUS: Análisis de Red término 'blockchain'}

El análisis de la literatura en base a las temáticas que cubre cada documento puede dar información sobre las características de la tecnología blockchain que se ven como potencial en cada área. Para ello se ha generado la matriz de adyacencia teniendo 
Figura 7. Análisis de red: publicaciones por temática.

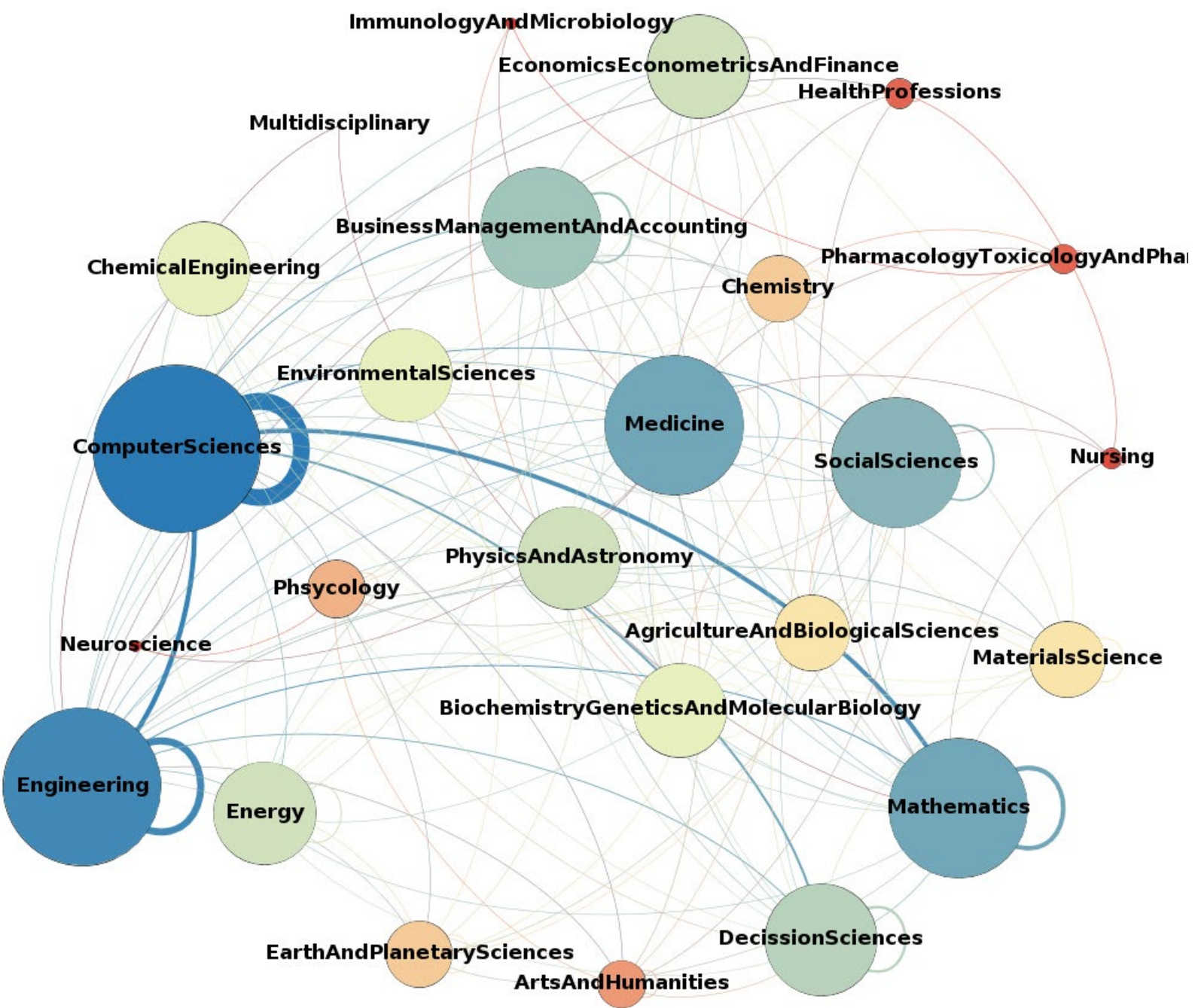

en cuenta las áreas propuestas por SCOPUS y su relación en base a cuantas de ellas están referidas a cada publicación. Este análisis inicial da como resultado una serie de gráficos que vislumbran la velocidad de expansión por temáticas y la multidisciplinaridad que presenta blockchain (Figura 7).

Los temas relacionados con el desarrollo de la propia tecnología aparecen como los de mayor área en el gráfico (mayor índice de centralidad Eigenvector). A simple vista la Figura 7 no presenta clústeres claros, indicando la interrelación entre los tipos de aplicación independientemente de área de utilización. Aplicando un algoritmo de agrupación este hecho se confirma, incluso si se eliminan las relaciones con las tres áreas principales: 'Computer Sciences', 'Engineering' y 'Mathematics'. Los enlaces entre los distintos nodos y su cercanía pueden expresar el modo de expansión de la tec- nología analizada entre temáticas que ya de por sí disponen de cierta interrelación.

Los datos de centralidad de las áreas más significativas se muestran en la Tabla I.

El índice de centralidad Eigenvector no solamente tiene en cuenta el peso de cada nodo y el número de vértices del que dispone, sino que es superior cuando los nodos con los que está relacionado disponen de un mayor número de vértices (constan de mayor importancia relacional). Por este motivo el orden de los temas cambia con respecto a la tabla que se obtiene ordenándolos simplemente por número de publicaciones. Los que están mejor posicionados, según el índice de centralidad, están relacionados con áreas en donde se están realizando labores que enlazan con más temáticas, bien sea porque su aplicación está más generalizada o 
Tabla I. Ordenación de las temáticas por centralidad Eigenvector

\begin{tabular}{|c|c|c|c|}
\hline Tema & $\begin{array}{l}\text { Centralidad } \\
\text { Eigenvector }\end{array}$ & Tema & $\begin{array}{l}\text { Centralidad } \\
\text { Eigenvector }\end{array}$ \\
\hline Computer Sciences & 0.31094288848437845 & Materials Science & 0.1906546087518685 \\
\hline Engineering & 0.30058870841135726 & $\begin{array}{l}\text { Biochemistry Genetics and } \\
\text { Molecular Biology }\end{array}$ & 0.18513246167342112 \\
\hline Mathematics & 0.2699913031336816 & $\begin{array}{l}\text { Agriculture and Biological } \\
\text { Sciences }\end{array}$ & 0.17480895796507104 \\
\hline Social Sciences & 0.2649111006515038 & Earth and Planetary Sciences & 0.16997807279373361 \\
\hline $\begin{array}{l}\text { Business Management and } \\
\text { Accounting }\end{array}$ & 0.2640410473564557 & Chemistry & 0.15445125142783628 \\
\hline Decission Sciences & 0.24816819182544514 & Phsycology & 0.13514595047386394 \\
\hline Medicine & 0.24815163023328568 & Arts and Humanities & 0.1350687183346808 \\
\hline Energy & 0.24051817074163126 & Health Professions & 0.09228017198030153 \\
\hline Physics and Astronomy & 0.2364377396591861 & Nursing & 0.06747375073154026 \\
\hline $\begin{array}{l}\text { Economics Econometrics and } \\
\text { Finance }\end{array}$ & 0.22844998034723527 & $\begin{array}{l}\text { Pharmacology Toxicology and } \\
\text { Pharmaceutics }\end{array}$ & 0.061721083781560125 \\
\hline Environmental Sciences & 0.22179096043638052 & Neuroscience & 0.0535144141999806 \\
\hline Chemical Engineering & 0.21977687155186879 & Multidisciplinary & 0.04398208714075491 \\
\hline
\end{tabular}

Figura 8. Matriz de adyacencia: 50 palabras clave de autor normalizadas con mayor ocurrencia.

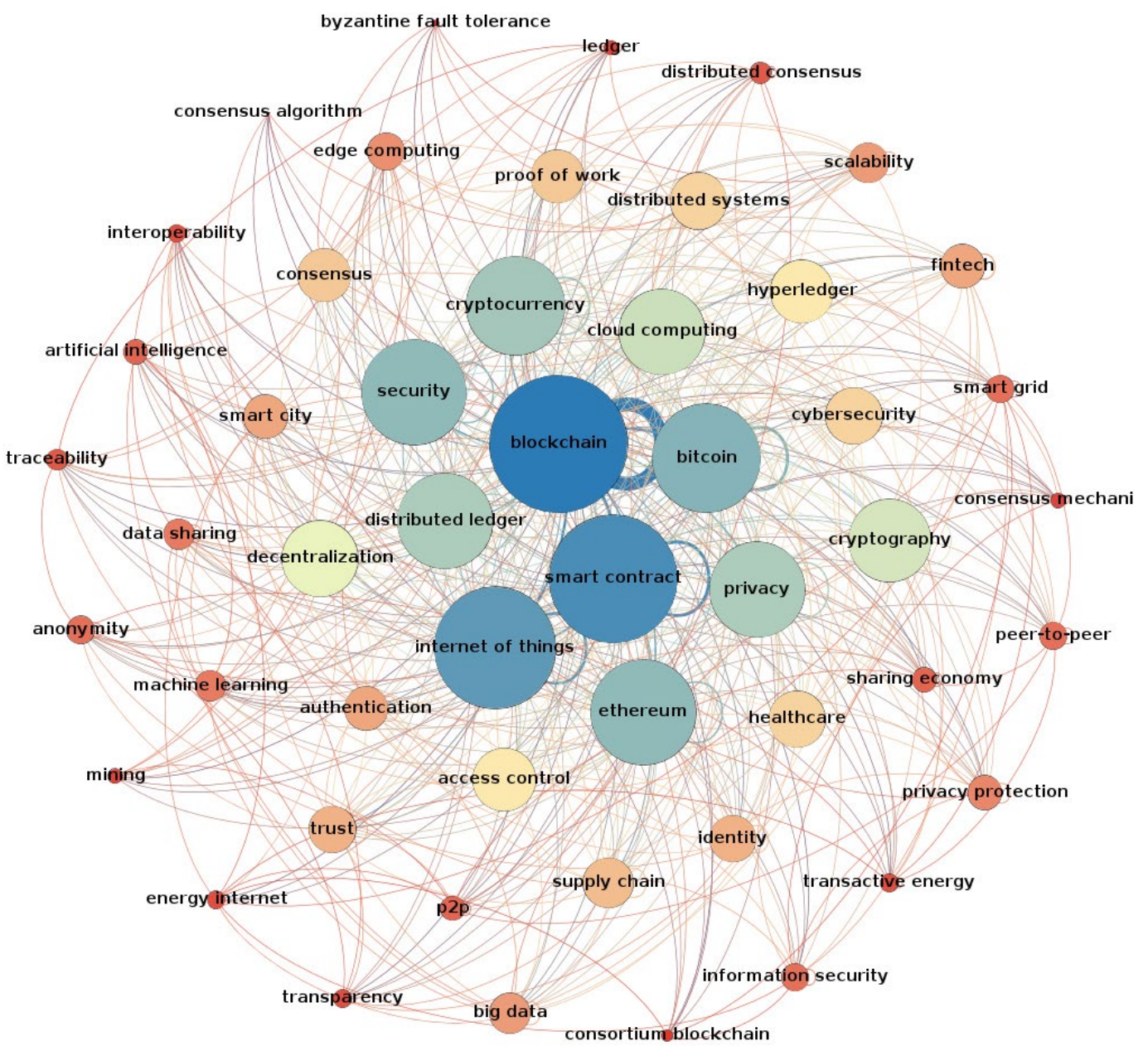


porque tiene que ver con desarrollos ligados a la base tecnológica empleados transversalmente.

Los temas con menor grado de centralidad pueden indicar una utilización de la tecnología blockchain más específica (se podría pensar que más novedosa) y quizás en un estado más preliminar o incipiente.

3.3.2 Palabras clave de autor normalizadas: Análisis de Red término 'blockchain'

Al disponer de un elevado número de palabras clave previamente normalizadas durante la fase de preparación de los datos, para que las gráficas sean más sencillas de analizar, se han representado las de mayor ocurrencia. El análisis de las palabras que cada autor considera claves en su publicación puede dar una visión global de los temas tratados dentro de cada trabajo y, además, mostrar los conceptos más importantes en la publicación. Su estudio también puede sacar a la luz temas de investigación emergentes y las nuevas tendencias en el desarrollo de la tecnología blockchain (Gutiérrez-Nieto y Serrano-Cinca, 2019).

El análisis de red realizado y representado en la Figura 8 es el resultado de la matriz de adyacencia generada tomando los 50 términos normalizados más significativos.

También se ha obtenido el grado de centralidad Eigenvector de la figura anterior, pudiéndose utilizar en futuros análisis, como indicador representativo y para la obtención de conclusiones.

3.3.3 Palabras clave de autor normalizadas: Análisis de Red - Clústeres

El estudio realizado de las palabras clave de autor presentes en la bibliografía permite obtener información sobre las nuevas líneas de investigación lanzadas en torno a un concepto en donde la tecnología blockchain está presente.

No se observan clústeres separados al aplicar técnicas de detección de agrupaciones. Incluso imponiendo restricciones al nivel de atracción aplicando técnicas como la de 'water leveling' (mayor que un número dado) no aparecen separaciones claras. Esto es debido a que todos los conjuntos de palabras clave comparten términos comunes relacionados principalmente con los elementos tecnológicos tractores como son 'blockchain' o 'smart contracts', o plataformas blockchain como 'Bitcoin' o 'Ethereum'.

La no existencia de agrupaciones puede indicar también que las aplicaciones tecnológicas de blockchain son compartidas entre todas las áreas de utilización. Esto ocurre cuando se habla de tecnologías transversales que afectan a fundamentos generales, alejándose de aplicaciones específicas orientadas exclusivamente a soluciones o nichos concretos.

Para poder identificar estas aplicaciones y nuevas tendencias del uso de la tecnología analizada por áreas, se han separado los datos filtrándolos por los términos de mayor peso en base a su número de apariciones e índice de centralidad. En cada subconjunto se ha vuelto a aplicar un análisis de red.

Este procedimiento permite realizar un 'zoom' dentro de un segmento específico y obtener información que orienta sobre la expansión, el posicionamiento y los temas de aplicación de la tecnología blockchain. Es una alternativa muy interesante cuando no existe una posibilidad de clusterización directa como se ha visto en este caso. Un ejemplo de ello se muestra en la Figura 9, la cual se ha generado al tomar el subconjunto de términos relacionados con una de las áreas de desarrollo con mayor número de centralidad Eigenvector: 'Internet of Things' (IoT).

Si dentro de este subconjunto se centra aún más la representación en los términos de más peso, aparecen con mayor definición los tipos de aplicación más utilizados en el área de IoT de la tecnología blockchain.

\subsection{Evaluación según temáticas: blockchain}

El análisis por temática indica claramente un gran crecimiento de aplicación de blockchain en multitud de áreas. El indicador de áreas temáticas queda cubierto con los datos obtenidos en la Tabla I. A su vez, la Figura 7 refleja el volumen de publicaciones por temática y la relación entre ellas. Los primeros puestos son los relacionadas con el desarrollo tecnológico de blockchain: computer sciences, engineering, y mathematics. Esto muestra que el tema que se está evaluando todavía se encuentra en fase de fuerte desarrollo a nivel tecnológico. Después aparecen las áreas con mayor desarrollo de publicaciones, destacan entre ellas con un índice de centralidad Eigenvector superior a 0,20 nueve sectores diferentes. Todas ellas contemplan un nivel de desarrollo suficiente para tenerlas en cuenta. No hay que perder de vista los documentos englobados en 'Multidisciplinary', con un nivel bajo de centralidad puede contener documentación de aplicaciones alternativas que pueden resultar más extrañas o novedosas.

Como indicadores interesantes en este caso para integrar en un cuadro de mando o aplicación de vigilancia tecnológica, se encontraría la tabla de centralidad (Tabla I) y la representación del análisis de red (Figura 7). 
Figura 9. Análisis de red: subconjunto 'IoT'

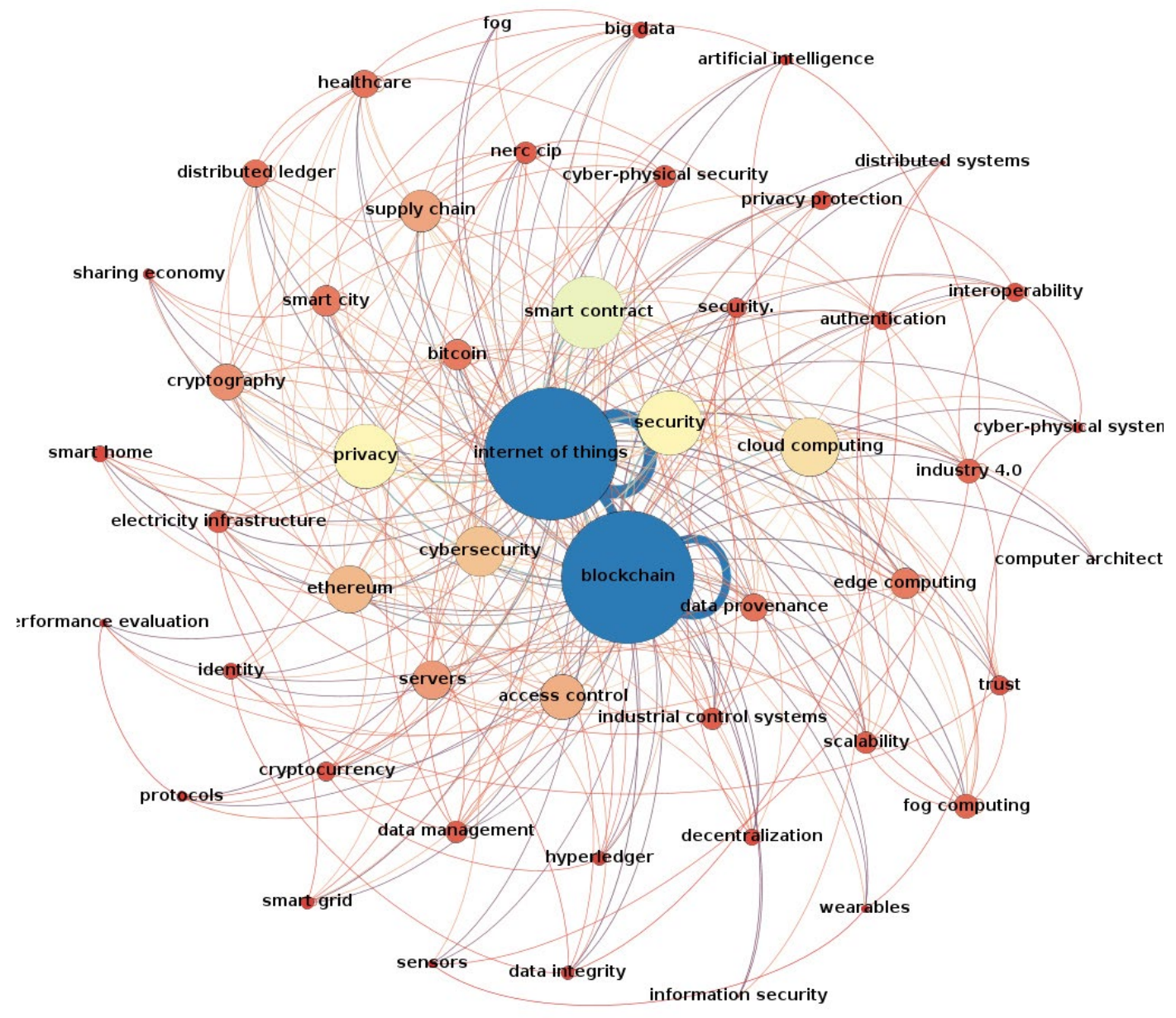

\subsection{Evaluación según palabras clave de autor normalizadas: blockchain}

Dentro de los análisis basados en palabras clave el término más empleado como cabe esperar es blockchain, dado que es la temática tecnológica central alrededor de la cual se centra nuestro estudio. Debajo de ella, tanto si se considera el número de aparición o el índice de centralidad Eigenvector aparecen dos términos clave: 'smart contract' y 'bitcoin', dos de las aplicaciones principales y potenciales de blockchain. Blockchain fue diseñado para hacer posible la primera criptomoneda confiable: bitcoin. Los contratos inteligentes están presentes en la evolución de blockchain (también denominada blockchain 2.0) (Müller y Hasic, 2016) en donde la red $\mathrm{BC}$ no solo está orientada a las transferencias económicas, sino que también es capaz de mantener y ejecutar las acciones acordadas en contratos programados (Bartoletti y Pompianu, 2017).

Uno de los términos de mayor relevancia, IoT, es una de las áreas de aplicación que escapan al mundo económico o contractual visto de un modo clásico, buscando la aplicación de las redes blockchain en el entorno del internet de las cosas. En este ámbito se comienza a ver la aplicación de esta tecnología para aportar características como seguridad, autonomía, confianza y colaboración, sin necesidad alguna de intermediación a todos los equipos conectados (Dorri y otros, 2016), añadiendo nuevas capacidades a los frameworks actuales utilizados (Ammar y otros, 2018). Existen trabajos de estudio de literatura que muestran el interés de la aplicación de blockchain en IoT, identificando usos lejos del caso de las criptomonedas, tales como (Conoscenti y otros, 2016):

- Gestión de datos y almacenamiento.

- Intercambio o comercio de bienes y datos.

- Gestión de identidades.

- Mecanismos de puntuación o reputación.

A continuación, en el ranking de palabras clave se muestran términos como 'security', 'privacy' y 'trust', que junto a 'decentralization' que aparece 
más adelante, son características propias de blockchain pudiendo ser aportadas al sistema que lo aplique. Si agrupamos aquellos casos que pertenecen a un ámbito cercano, los campos de aplicación que han publicado más trabajos han sido los relacionados con:

- Control de accesos y autenticación.

- Cadena de suministro.

- Computación en la nube, computación 'Edge' y Big Data.

- Fintech.

- Salud.

- Smart Grid y energía.

- Machine learning e Inteligencia artificial.

- Economía colaborativa (sharing economy).

Los elementos identificados son comparables a los mostrados en estudios bibliométricos realizados sobre esta tecnología (Casino y otros, 2019) por lo que las conclusiones obtenidas durante la fase de evaluación se encuentran alineadas con otras fuentes.

La identificación de las áreas de desarrollo y aplicación de esta tecnología en un sector determinado, incluyéndolo dentro de los procesos de vigilancia tecnológica, permite disponer de una visión dinámica de la evolución de la misma. Incorporar estos datos dentro del cuadro de gestión puede servir como elemento en la toma de decisiones a la hora de adoptar una nueva tendencia o de invertir en ella, marcando la diferencia frente a competidores.

\section{CONCLUSIONES}

La velocidad de desarrollo de nuevas tecnologías crece cada vez más rápido. Este hecho trae nuevas oportunidades y amenazas a las diferentes organizaciones. Su adopción en muchos casos es clave para poder mantener la competitividad. El ser capaces de detectarlas tempranamente mejora las posibilidades de adaptarse a los nuevos cambios y de aprovechar las nuevas oportunidades que aparecen.

La vigilancia tecnológica no es algo sencillo de llevar a cabo y menos cuando se habla de tecnologías aun no consolidadas. Los nuevos desarrollos se mantienen en secreto y no salen a la luz hasta que una empresa dispone de un producto comercializable. En ese momento dicha organización ha aprovechado ya su ventaja competitiva llevando por delante un tiempo considerable a su competencia, que debe adaptarse a los nuevos avances a partir de ese momento.

Hoy en día, la mayor parte de estos avances científico-tecnológicos se encuentran reflejados en publicaciones académicas, bien sean artículos de revista o los presentados en congresos. A su vez existen importantes servicios que publican bases de datos recopilados de los mismos, constituyendo un conjunto detallado y procesable de la información contenida en ellos. Sitios webs o motores de búsqueda de publicaciones científicas tales como 'Web of Science', SCOPUS o Google Scholar son ampliamente reconocidas como fuentes confiables y objetivas. Reúnen no solo artículos o papers de revistas científicas, sino también artículos de congresos en donde se tratan temas todavía no plenamente conocidos o establecidos.

Existen herramientas para la realización de estudios bibliométricos que analizan retrospectivamente la producción académica, siendo el investigador el que debe sacar sus propias conclusiones. La incorporación de nuevas utilidades, complementarias a las anteriores, que permitan extraer nuevas tendencias en una fase temprana, pueden ser de gran ayuda para abordar nuevas investigaciones.

Estos motivos han llevado al desarrollo de la metodología descrita a lo largo del documento, adaptando diferentes técnicas existentes hoy en día para conseguir el objetivo de obtener, del modo más automatizado y objetivo posible, indicadores que muestran el nivel de desarrollo de nuevas tecnologías emergentes y sus posibles áreas de aplicación. El análisis de los datos en base a mecanismos ampliamente utilizados en el campo de 'Data Analytics' o en KDD, como es el caso de CRISP-DM (Chapman y otros, 2000), marca las pautas de sistematización del proceso, posibilitando la definición y extracción de indicadores integrables dentro de los elementos de vigilancia tecnológica. Con el objetivo final de que los resultados sirvan como fuente de innovación, inteligencia de negocio, o como factor adicional de toma de decisiones estratégicas.

La Ciencia se nutre del conocimiento existente generado por los investigadores, gran parte presente en publicaciones académicas, sobre el que se desarrolla y crece. Pero para ponerlo en valor es necesario dotar de pragmatismo a las líneas que emergen de las nuevas investigaciones.

Los datos empleados a lo largo de este trabajo han sido recogidos desde SCOPUS por ser la fuente más fácilmente automatizable desde el punto de vista de los autores, en términos de sencillez de obtención y procesamiento de los mismos. Aun así, la información descargada no puede considerarse completamente estructurada. Su contenido se extrae de la narrativa de los autores y de campos específicos que ellos mismos completan. A parte de no encontrarse normalizados, están expresados en lenguaje natural, por este motivo se ha propuesto emplear técnicas empleadas en análisis de redes y procesado del lenguaje natural. 
Como caso de aplicación se ha utilizado 'blockchain', una de las tecnologías que, a pesar de haberse creado para un objetivo en concreto, se ha convertido en una de las más disruptivas y de aplicación transversal en nuestros días. Blockchain fue diseñada para hacer realidad la primera criptomoneda confiable (Bitcoin), pero después su aplicación ha ido creciendo y extendiéndose a lo largo de multitud de sectores y áreas. Llegando a tal punto que, en muchos casos, es una cuestión de identificar el cómo aplicarla a un nuevo sector para que marque la diferencia en el mismo. Este es un caso en el que es necesario vigilar su expansión e idear nuevos modos de utilización para obtener ventajas competitivas en nuestro entorno. Este argumento se ha visto reforzado con los resultados obtenidos sobre el número de publicaciones y su evolución por temáticas. El gran número de artículos de conferencias frente al de artículos publicados en revistas deja entrever que se está tratando con un concepto novedoso sobre el que se están desarrollando muchos trabajos que, posiblemente, se conviertan en futuros casos de uso.

La metodología desarrollada aplicada a la literatura existente durante el periodo 2000-2018 del término 'blockchain' ha permitido posicionar el estado actual del mismo. Como resultado, se han concluido las diferentes tendencias y su próxima evolución, así como las áreas más maduras y los campos donde su desarrollo es más incipiente. Este estudio ha permitido identificar las tendencias tecnológicas del uso de blockchain fuera de las aplicaciones consideradas clásicas como son las criptomonedas y sus usos dentro del mundo de la banca o las llamadas 'fintechs'. Por tratarse de aplicaciones clásicas, se han dejado fuera del alcance del análisis.

El objetivo de realizar un proceso sistemático, automatizable y repetible ha tomado como referencia la metodología CRISP-DM (Chapman y otros, 2000), adaptándola a las características de la información a tratar y objetivos a conseguir.

La aplicación de análisis de red, primero sobre la clasificación temática propuesta por SCOPUS, y más adelante sobre las palabras clave de autor, ha permitido inferir los casos de aplicación según su importancia, tomando como referencia indicadores como el índice de centralidad. Como resultado se han identificado aquellas áreas donde la adopción de blockchain está aportando ventajas claras.

Este enfoque relacional ha dado lugar a algoritmos aplicables de un modo periódico para observar las tendencias y evolución de la tecnología blockchain. Esto posibilita la identificación de nuevas aplicaciones o su expansión a nuevas áreas temáticas cuando comiencen a aparecer publicaciones sobre ellas. Los mecanismos empleados para con- cluir las aplicaciones con mayor potencial relacionadas con las palabras clave de mayor peso, ayudan a profundizar sobre el modo de utilización de esta tecnología en sus diferentes vertientes.

La no detección de clústeres dentro del análisis de red realizado sobre los términos clave, es probablemente debido a la característica de transversalidad de esta tecnología en cuanto a aplicación, mostrando cómo hay una relación fuerte de los conceptos nucleares de blockchain y las áreas en donde se está desarrollando.

Para profundizar en el estudio se ha realizado un análisis de subconjuntos de documentos relacionados con temática similar, eliminado el peso de las palabras clave que hacen referencia a la propia tecnología. Esto ha permitido centrar el tipo de aplicaciones blockchain en aquellas áreas que están mostrando mayor interés, sacando a la luz aquellos sectores que están invirtiendo un gran esfuerzo en el estudio de la aplicación de esta tecnología por sus capacidades o propiedades. Tal es el caso de IoT (Internet of Things), en la cual blockchain se presenta como parte clave en la formación de dispositivos colaborativos y seguros. Sus capacidades son de gran valor en los desarrollos de sistemas en la nube, relacionados con el Big Data y en los procesos de 'machine learning'. Todas ellas tecnologías sobre las que se están basando nuevos modelos de negocio y en donde blockchain puede aportar su propuesta de valor.

Merece la pena plantear para un próximo desarrollo la inclusión de nuevos indicadores en la fase de objetivos, e incluir dentro del análisis también las temáticas que se han considerado como residuales debido a que ahí se pueden encontrar aplicaciones más novedosas o alternativas.

También queda pendiente la automatización del análisis de abstracts e inclusión de nuevas características a los datos que puedan aportar ventajas a la hora de vigilar las tecnologías emergentes.

Al realizar una vigilancia de este tipo de manera periódica y automática a lo largo de los años, se podrán ver las nuevas tendencias e identificar los campos donde se estén invirtiendo los mayores esfuerzos en la tecnología objetivo.

En la actualidad, la innovación es un concepto íntimamente vinculado con la competitividad, siendo a su vez el fruto de un proceso que comienza con la Investigación Básica. Los autores del artículo consideramos que el acortamiento del proceso de innovación requiere de un enfoque de Investigación Básica Orientada, siendo coherente con el desarrollo de metodologías que complementen a las tradicionales de análisis bibliométrico aportando un enfoque hacia líneas de investigación de futuro. 


\section{REFERENCIAS}

Aguillo, I. F. (2012). Is Google Scholar useful for bibliometrics? A webometric analysis. Scientometrics, 91(2), 343-351. https://doi.org/10.1007/s11192-011-0582-8

Ahram, T., Sargolzaei, A., Sargolzaei, S., Daniels, J., Amaba, B. (2017). Blockchain technology innovations. 2017 IEEE Technology \& Engineering Management Conference (TEMSCON), 137-141. https://doi. org/10.1109/TEMSCON.2017.7998367

Alves, A. D., Yanasse, H. H., Soma, N. Y. (2016). An analysis of bibliometric indicators to JCR according to Benford's law. Scientometrics, 107(3), 1489-1499. https://doi.org/10.1007/s11192-016-1908-3

Ammar, M., Russello, G., Crispo, B. (2018). Internet of Things: A survey on the security of IoT frameworks. Journal of Information Security and Applications, 38, 8-27. https://doi.org/10.1016/j.jisa.2017.11.002

Bartoletti, M., Pompianu, L. (2017). An Empirical Analysis of Smart Contracts: Platforms, Applications, and Design Patterns. En M. Brenner, K. Rohloff, J. Bonneau, A. Miller, P. Y. A. Ryan, V. Teague, A. Bracciali, M. Sala, F. Pintore, y M. Jakobsson (Eds.), Financial Cryptography and Data Security 10323, 494-509). Springer International Publishing. https://doi.org/10.1007/978-3-319-70278-0_31

Berka, P., Rauch, J. (2014). Machine Learning and Association Rules. 31.

Bollinger, A. S., Smith, R. D. (2001). Managing organizational knowledge as a strategic asset. Journal of Knowledge Management, 5(1), 8-18. https://doi. org/10.1108/13673270110384365

Bontis, N., Nikitopoulos, D. (2001). Thought leadership on intellectual capital. Journal of Intellectual Capital, 2(3), 183-191. https://doi.org/10.1108/14691930110400182

Boucher, P., Nascimento, S., Kritikos, M. (2017). How blockchain technology could change our lives (PE 581.948). EPRS European Parlamientary Research Service.

Casino, F., Dasaklis, T. K., Patsakis, C. (2019). A systematic literature review of blockchain-based applications: Current status, classification and open issues. Telematics and Informatics, 36, 55-81. https://doi. org/10.1016/j.tele.2018.11.006

Chapman, P., Clinton, J., Kerber, R., Khabaza, T., Reinartz, T., Shearer, C., Wirth, R. (2000). Step-by-step data mining guide. 76.

Chartered Accountants. (2016). The Future of Blockchain: Aplications and implications of distributed ledger technology. Deloitte. http://charteredaccountantsanz. com/futureinc

Conoscenti, M., Vetro, A., De Martin, J. C. (2016). Blockchain for the Internet of Things: A systematic literature review. 2016 IEEE/ACS 13th International Conference of Computer Systems and Applications (AICCSA), 1-6. https://doi.org/10.1109/AICCSA.2016.7945805

Conte de Leon, D., Stalick, A. Q., Jillepalli, A. A., Haney, M. A., Sheldon, F. T. (2017). Blockchain: Properties and misconceptions. Asia Pacific Journal of Innovation and Entrepreneurship, 11(3), 286-300. https://doi. org/10.1108/APJIE-12-2017-034
Dorri, A., Kanhere, S. S., Jurdak, R. (2016). Blockchain in Internet of Things: Challenges and Solutions. 13.

Dr Garrick Hileman, Michel Rauchs. (2017). Global Blockchain Benchmarking Study. Cambridge Centre for Alternative Finance, University of Cambridge. https:// doi.org/10.2139/ssrn.3040224

Edvinsson, L. (2013). IC 21: Reflections from 21 years of IC practice and theory. Journal of Intellectual Capital, 14(1), 163-172. https://doi. org/10.1108/14691931311289075

Fayyad, U. Piatetsky-Shapiro, G.; Smyth, P. (1996). From Data Mining to Knowledge Discovery in Databases. American Association for Artificial Intelligence, 18 .

Gutiérrez-Nieto, B.; Serrano-Cinca, C. (2019). 20 years of research in microfinance: An information management approach. International Journal of Information Management, 47, 183-197. https://doi.org/10.1016/j. ijinfomgt.2019.01.001

Harzing, A.-W.; Alakangas, S. (2016). Google Scholar, Scopus and the Web of Science: A longitudinal and cross-disciplinary comparison. Scientometrics, 106(2), 787-804. https://doi.org/10.1007/s11192015-1798-9

Kogabayev, T.; Maziliauskas, A. (2017). The definition and classification of innovation. HOLISTICA - Journal of Business and Public Administration, 8(1), 59-72. https://doi.org/10.1515/hjbpa-2017-0005

López-Robles, J. R. ; Otegi-Olaso, J. R., Porto Gómez, I. ; Cobo, M. J. (2019). 30 years of intelligence models in management and business: A bibliometric review. International Journal of Information Management, 48, 22-38. https://doi.org/10.1016/j.ijinfomgt.2019.01.013

Michalisin, M. D., Smith, R. D.; Kline, D. M. (1997). In search of strategic assets. The International Journal of Organizational Analysis, 5(4), 360-387. https://doi. org/10.1108/eb028874

Müller, C.; Hasic, D. (2016). Blockchain: Technology and Applications.

North, K.; Kumta, G. (2018). Knowledge Management. Springer International Publishing. https://doi. org/10.1007/978-3-319-59978-6

Opsahl, T., Agneessens, F.; Skvoretz, J. (2010). Node centrality in weighted networks: Generalizing degree and shortest paths. Social Networks, 32(3), 245-251. https://doi.org/10.1016/j.socnet.2010.03.006

Sveiby, K. E. (1997). The new organizational wealth: Managing \& measuring knowledge-based assets (1st ed). Berrett-Koehler Publishers.

Xi, S. M.; Cho, Y. I. (2012). Comparison of Application Effect of Natural Language Processing Techniques for Information Retrieval. Journal of Institute of Control, Robotics and Systems, 18(11), 1059-1064. https:// doi.org/10.5302/J.ICROS.2012.18.11.1059

Zinoviev, D. (2018). Complex Network Analysis in Python. The Pragmatic Programmers, LLC. 